\title{
Ground Improvement: Referees 2018
}

The following is a list of referees who have reviewed papers for Ground Improvement between 1 December 2017 and 30 November 2018. The Institution of Civil Engineers is very grateful for their assistance.

We are continually looking for suitable reviewers for papers submitted to Ground Improvement. Papers published in the Proceedings of the ICE must be submitted to at least two independent referees to judge accuracy, style, impact, importance and interest.

If you are interested in reviewing articles on any topic related to ground improvement, please submit your name, qualifications or $C V$, and areas of

Ahmad Safuan A Rashid Pouyan Abbasi maedeh

Azneb Abdul Salam

Abiola Abiodun

Hadi Ahmadi

Md Ashraf Ahmed

Aly Ahmed

Santiago Alija Sanchez

Costas Anagnostopoulos

Panagiotis Andreou

Arul Arulrajah

Afshin Asadi

Mousa Attom

Adekemi Ayodele

Mustafa Aytekin

Aritra Banerjee

Pranjal Barman

Sudip Basack

Marina Bellaver Corte

Mounir Bouassida

Chris Bridges

Malcolm Burbank

Rafaela Cardoso

Jorge Castro

Jinchun Chai

Sayantan Chakraborty

Cheeming Chan

Qingsheng Chen

Liang Cheng

Prinya Chindaprasirt

Annamaria Cividini

Nilo Consoli

Stephen Corbet

Nuno Cristelo

Olivier Cuisinier

Jonathan Daramalinggam

Champakali DAS

Kousik Deb
An Deng

Mahdi Disfani

Yan-Jun Du

Magdi El-Emam

Vítor Faro

Wissem frikha

Padmanabhan G

Sridhar Gangaputhiran

Sara Gargano

Meixiang Gu

Jun Guo

Aliasger Haiderali

Jie Han

Asadul Haque

Michael Harbottle

Kimitoshi Hayano

Ana Heitor

Michiel Heyns

Chu Ho

Syed Khaja Hussaini

Pitthaya Jamsawang

C J F P Jones

Anil Joseph

Ashish Juneja

José Justo

Shaymaa Kadhim

Doncho Karastanev

Richard Kelly

Houssam Khelalfa

Prabir Kolay

Leena Korkiala-Tanttu

Dharmesh Lal

Chun Fai Leung

LiHui Li

Stefania Lirer

Andres Lotero

David Lourenço

Yitian Lu expertise. We are in need of individuals who will agree to review papers in a timely fashion (within 3 to 4 weeks of receipt) and provide confidential feedback to the Editorial Advisory Panel concerning the quality of the paper and any suggested revisions that would be appropriate.

If you are such a person, please contact Cherise Lopes-Baker (tel.: +44 (0)207 665 2249; e-mail: cherise.lopes-baker@icepublishing.com) for more information on the referee process.
Jianqin Ma

Sina Majidian

Mona Mansour

Sérgio Marques

Bryan McCabe

Martin Mgangira

Bashir MIR

Mehdi Mirzababaei

Mostafa Mohamed

Yahia Mohamedzein

Brina Montoya

Yasser Mostafa

Sravan Muguda Viswanath

A Murali Krishna

Ngoc Trung Ngo

Thanh Nguyen

Sanjay Nimbalkar

Makan Noorbakhsh

Jéssica Novaes

Dominic Ong

Philip Paige-Green

Loizos Pelecanos

Guilherme Pepplow Piuzzi

Steve Perkins

M Isabel M Pinto

Supasit Pongsiwasathit

H G Poulos

Ananthakrishna Prabhu Hindusthan

Akash Priyadarshee

Anand J Puppala

Yafei Qiao

Sujatha Ramani

Alireza Rezagholilou

Sara Rios

Cholachat Rujikiatkamjorn

Cesar Ruver

David Saftner

Marina Schnaider Bortolotto
Andrea Segalini

Colin Serridge

Brian Sexton

Ehsan Seyedi Hosseininia

Yazdan Shams Maleki

Anil Sharma

Sailesh Shrestha

Raj Siddharthan

Carina Silvani

Baleshwar Singh

T G Sitharam

Vandana Sreedharan

Anubhav Srivastav

Daisuke Suetsugu

Xiaohui Sun

Hidenori Takahashi

Jiro Takemura

Yong Tan

Rui Tomasio

Ikuo Towhata

Laricar Dominic Trani

J S Vinod

Jingan Wang

Jun Wang

Peng Wang

Shanyong Wang

Huai-na Wu

Ye-Shuang Xu

Fang $\mathrm{Xu}$

Xiaoming Yang

Haitao Yu

Yiping Zhang

Minghua Zhao

Haizuo Zhou

Seyed Mohammad Zomorodian 